\title{
ON HAAR'S THEOREM CONCERNING CHEBYCHEV APPROXIMATION PROBLEMS HAVING UNIQUE SOLUTIONS ${ }^{1}$
}

\author{
JOHN C. MAIRHUBER
}

1. Introduction. A. Haar [5] discussed the Chebychev approximation problem in the following setting. Let $M$ be a compact set in euclidean $k$-dimensional space $E_{k}$, such that $M$ contains at least $n$ distinct points, $n$ a given natural integer. Let $f_{1}(x), \cdots, f_{n}(x)$ be $n$ real continuous functions defined on $M$. Let $f(x)$ also be given as a real continuous function on $M$. A best approximation to $f(x)$ by $f_{1}(x), \cdots, f_{n}(x)$ in the sense of Chebychev is a linear combination

$$
\sum_{\nu=1}^{n} a_{\nu}^{*} f_{v}(x)
$$

such that

$$
\max _{x \in M}\left|f(x)-\sum_{\nu=1}^{n} a_{\nu}{ }^{*} f_{\nu}(x)\right| \leqq \max _{x \in M}\left|f(x)-\sum_{\nu=1}^{n} a_{\nu} f_{\nu}(x)\right|
$$

for every set of real numbers $\left(a_{1}, \cdots, a_{n}\right)$. The existence of such best approximations is well known, and can readily be established by means of familiar continuity and compactness arguments. In his paper, Haar investigated the question of the unicity of the solution, and obtained the following.

THEOREM 1. A necessary and sufficient condition that a unique solution $\left(a_{1}{ }^{*}, \cdots, a_{n}{ }^{*}\right)$ exist for every given $f(x)$ real and continuous on $M$ is that every linear combination

$$
a_{1} f_{1}(x)+\cdots+a_{n} f_{n}(x) \quad\left(a_{1}, \cdots, a_{n}\right) \neq(0, \cdots, 0),
$$

vanishes in at most $n-1$ distinct points of $M$.

It is easy to see that the linear combinations (1) vanish in at most $n-1$ distinct points of $M$ if and only if the set of functions

$$
f_{1}(x), \cdots, f_{n}(x)
$$

enjoys the following property.

Received by the editors June 23, 1955.

${ }^{1}$ This work was performed under an Air Force contract at the University of Pennsylvania. 
Property D.

$$
\left|\begin{array}{cccc}
f_{1}\left(x_{1}\right) & f_{2}\left(x_{1}\right) & \cdots & f_{n}\left(x_{1}\right) \\
f_{1}\left(x_{2}\right) & f_{2}\left(x_{2}\right) & \cdots & f_{n}\left(x_{2}\right) \\
\vdots & \vdots & & \vdots \\
f_{1}\left(x_{n}\right) & f_{2}\left(x_{n}\right) & \cdots & f_{n}\left(x_{n}\right)
\end{array}\right| \neq 0
$$

for every set of $n$ distinct points $x_{1}, \cdots, x_{n}$ of $M$.

There are well known concrete examples of sets of functions $f_{v}(x)$ enjoying property $\mathrm{D}$ in the special case where $M$ is either a finite closed interval of the real line or else the circumference of a circle. See S. Bernstein [2], where such sets of functions are studied and called by him Chebychev systems. The question naturally arises as to what other compact subsets $M$ of euclidean spaces may serve as domains of definition of sets of functions $f_{1}(x), \cdots, f_{n}(x)$ enjoying the property $\mathrm{D}$.

At the end of his paper, Haar mentions that $M \subset E_{k}$ may contain no interior points for $k \geqq 2$. This is indeed clear, for if $x_{1}$ and $x_{2}$ are points of an open sphere $S$ of $M$, then $x_{1}$ and $x_{2}$ may interchange their positions in $M$ by a continuous simultaneous motion within $S$, without ever coinciding with one another or any of the points $x_{3}, \cdots, x_{n}$, which are held fixed. However, the result of this interchange is that the determinant (2) has changed sign, and must therefore have vanished for some intermediate position of $x_{1}$ and $x_{2}$ in violation of the property $D$. Haar's remark provides a necessary but by no means sufficient property of the set $M$.

The main result of this paper is that in order that the functions $f_{1}(x) \cdots, f_{n}(x)$ satisfy the condition of Haar's theorem, where $n \geqq 2$, the domain of definition $M$ of the functions is restricted to be the homeomorphic image of a closed subset of a finite interval, with the possible exception, when $n$ is even and $M$ is connected, that $M$ may be the homeomorphic image of the circumference of a circle. The result is summarized in

Theorem 2. A compact subset $M$ of $E_{k}$ containing at least $n$ points, $n \geqq 2$, may serve as the domain of definition of a set of real continuous functions $f_{1}(x), \cdots, f_{n}(x)$ having property $\mathrm{D}$, if and only if $M$ is homeomorphic to a closed subset of the circumference of a circle.

The proof is obtained by means of an auxiliary theorem of interest in itself.

TheORFM 3. $A$ compact set $M$ in $E_{n}$, having the property that at 
most $n$ points of $M$ lie on any hyperplane of $E_{n}$, is the homeomorphic image of a closed subset of the circumference of a circle.

The author wishes to express his sincere appreciation to Professor I. J. Schoenberg for having suggested the problem, and for his very kind assistance in its solution.

2. Characterization of a set in $E_{n}$ homeomorphic to $M$. Let $S$ be the set of points in $E_{n}$, whose coordinates are

$$
\left(f_{1}(x), f_{2}(x), \cdots, f_{n}(x)\right), \quad x \in M \text {. }
$$

The mapping $\phi$ of $M$ onto $S$ is continuous, since each of the $f_{v}(x)$ $(\nu=1,2, \cdots, n)$ is continuous. Furthermore, $\phi$ is one to one, since if for every $\nu(\nu=1,2, \cdots, n)$

$$
f_{\nu}\left(x_{1}\right)=f_{\nu}\left(x_{2}\right), \quad x_{1}, x_{2} \in M,
$$

the determinant (2) vanishes. Inasmuch as $M$ is compact, $\phi$ is a homeomorphism.

The set $S$ can be characterized by the following properties.

(i) $S$ is a compact set of at least $n$ points;

$$
S \subset E_{n}=\left(y_{1}, \cdots, y_{n}\right) \text {. }
$$

(ii) An arbitrary hyperplane passing through the origin $o$,

$$
a_{1} y_{1}+a_{2} y_{2}+\cdots+a_{n} y_{n}=0, \quad \sum_{v=1}^{n} a_{v}^{2}>0,
$$

can meet $S$ in at most $n-1$ points. Consequently, for $1 \leqq m \leqq n-1$, at most $m$ points of $S$ can lie on the same $m$ dimensional flat of $E_{n}$ containing $o$. In particular, $o$ cannot belong to $S$.

(iii) Since the determinant (2) does not vanish, it follows from a well-known theorem of Fenchel [4] that the point $o$ cannot be contained in the convex hull of any one component of $S$.

(iv) The point $o$ cannot be in the convex hull of any $n$ components of $S$, by an extension of Fenchel's theorem obtained by L. N. H. Bunt [3].

3. Characterization of a set in $E_{n-1}$ homeomorphic to $M$. We now use the following theorem of Hanner and Rådström [6].

Theorem 4. Let $M$ be a compact set in $E_{n}$, and let $A$ be a closed subset of $M$. Let $p$ be a point of $E_{n}$ which is not in the convex hull of any $n$ points of $M$, and which is outside the convex hull of $A$. Then there is a hyperplane $\pi$ through $p$ which meets no point of $M$ or of the convex hull of $\boldsymbol{A}$. 
By (iv) above, there is a hyperplane $\pi^{\prime}$ passing through $o$ such that no point of $S$ lies on $\pi^{\prime}$. Since $S$ is compact, and $o$ is not a point of $S$, there is a hyperplane $\pi$ parallel to $\pi^{\prime}$ such that $\pi$ contains neither $o$ nor any points of $S$.

Let $T$ be the set obtained by projecting $S$ on $\pi$ through $o$. No two points of $S$ can project into the same point of $T$ by property (ii) above. Hence $T$ is a continuous, one to one image of a compact set, so that $T$ is homeomorphic to $S$, and hence to $M$.

For simplicity in the sequel, we characterize the set $T$ of $E_{n-1}$ as a subset $T$ of $E_{m}$. From (ii) we have

(v) $T$ is a compact set in $E_{m}, m=n-1$, such that at most $m$ points lie on the same hyperplane of $E_{m}$.

The result stated in Theorem 2 now follows from the proof of Theorem 3. That the set $T$ is one-dimensional follows from Theorem 10 in the paper of Nöbeling cited in the bibliography; this also follows from the fact that the set $T$ has the property that any two points of $T$ are separated by $m$ points [7].

\section{Proof of Theorem 3.}

Definition 1. A continuum $C$ in $E_{n}$ is said to be of bounded order if no hyperplane meets $C$ in more than some fixed number $k$ of points.

Definition 2. A point of ramification is a point common to three or more simple arcs, which pairwise have no other common point.

The following theorem is taken from two theorems proved by Marchaud [8].

THEOREM 5. Let $M$ be a continuum of bounded order in $E_{n}$. Then $M$ can be decomposed into a denumerable infinity of simple rectifiable arcs, such that any two of them have only a finite number of points in common. If there are no points of ramification, then the continuum is a simple rectifiable Jordan curve, open or closed.

Marchaud further shows that a plane continuum of order two and a three-dimensional continuum of order three have no point of ramification. Generalizing the proof to order $m$, we obtain

Theorem 6. A continuum of order $m$ in $E_{m}$ has no point of ramification.

Proof. Suppose that $q$ is a point of ramification of a continuum $C$ of $E_{m}$, where $C$ is of order $m$. Let $q a, q b$, and $q c$ be three arcs having in common the point $q$, but otherwise pairwise disjoint. On the arc $q a$, pick $m-1$ points $a_{1}, \cdots, a_{m-1}$, distinct from one another and from $q$. These points cannot lie on a flat of dimension less than $m-1$, and hence, together with $q$, determine a hyperplane $\pi$ containing $m$ 
points of $M$. One of these points, say $a_{1}$, has the property that no points of the arc $q a_{1}$ other than $q$ and $a_{1}$ lie on the plane $\pi$. With the exception of the points $q$ and $a_{1}$, at least two of the arcs $q a_{1}, q b$, and $q c$ lie entirely on the same side of $\pi$. For simplicity call these arcs $\alpha$ and $\beta$. Let $p_{\nu}$ be a sequence of points on $\alpha$ such that

$$
\lim _{\nu \rightarrow \infty} p_{\nu}=q .
$$

Each point $p_{\nu}$ together with $a_{1}, a_{2}, \cdots, a_{m-1}$ determines a hyperplane $\pi_{\nu}$ containing $m$ points of $M$. Let $\pi_{\nu}$ have equation

$$
f^{(\nu)}(x)=a_{1}^{(\nu)} x_{1}+\cdots+a_{m}^{(\nu)} x_{m}+a_{m+1}^{(\nu)}=0,
$$

where the coefficients can be so chosen that

$$
f^{(\nu)}(q)>0 \text {. }
$$

Let $r$ be a point of $\beta$ distinct from $q$. Then if $f^{(\nu)}(r) \leqq 0$ for some $\nu$, there are $m+1$ points on $\pi_{\nu}$. This, however, must be the case, since $f^{(\nu)}(r)>0$ for all $\nu$ would contradict the fact that $\alpha$ and $\beta$ are on the same side of $\pi$.

A convex curve in $E_{m}$ is defined to be a Jordan curve having at most $m$ points on any $m-1$ dimensional hyperplane. From Theorems 5 and 6 , it follows that the set $T$ has components which are single points or convex curves.

An $m$-dimensional convex curve can be closed only when $m$ is even. For $m$ points on the curve determine a hyperplane, and if none of these points is an endpoint of the arc, the arc must cross the hyperplane at each of these $m$ points [8]. Thus the curve can be closed only when the number of crossings is even, that is, when $m$ is even. In the event that $T$ is connected, the results obtained show that $T$ is an open or closed convex curve, and thus that $T$ is the homeomorphic image of a closed subset of a circle.

If $m$ is even, and $T$ consists of more than one component, then no nontrivial component $\tau$ of $T$ is a closed Jordan curve. For assume otherwise. Select any $m-1$ points on $\tau$, and let $p$ be a point not on $\tau$. These $m$ points determine a hyperplane $\pi$ meeting $\tau$ in $m-1$ points.

There are now two possibilities. In the first case, if $\tau$ crosses $\pi$ at each of the $m-1$ points in which it meets $\pi$, then there is an $m$ th point in which $\tau$ crosses $\pi$, giving us $m+1$ points on $\pi$, a contradiction. In the second case, suppose that at some point $a$ common to $\tau$ and $\pi, \tau$ does not cross $\pi$. Then by the argument used in the proof of Theorem 6 , displacing the point $a$ slightly, we obtain $m$ points in which $\tau$ meets $\pi$, and arrive at a contradiction as before. 
Moore and Kline [9] proved the following.

THEOREM 7. In order that a compact point set $N$ in $E_{2}$ should be a subset of a simple continuous arc, it is necessary and sufficient that every component of $N$ should be either a single point or a simple continuous arc $\tau$ such that no point of $\tau$ other than an endpoint should be a limit point of $[N-\tau]$.

Zippin [12] has investigated the extension of this theorem to other spaces. For a space $C$ which is complete, metric, separable, connected and locally connected, the following conditions are found to be equivalent, and either of them is a necessary and sufficient condition that the Moore-Kline theorem hold in $C$.

Condition 1. If $b$ is an endpoint of an arc $\tau$ of $C$, then for every preassigned $\epsilon>0$, there exists a $\delta>0$ such that if $y$ and $z$ are points of $(C-\tau) \cdot S(b, \delta)$, the set $(C-\tau) \cdot S(b, \epsilon)$ contains an arc $y z$.

Condition 2. Let $R$ be a region in $C$, and $a b$ be an arc in $C$ such that $a$ is on the frontier of $R$ and $[a b-a] \subset R$. Then $R-[a b-a]$ is connected.

For $n \geqq 3$, Condition 1 is established by the result that an $n$-sphere cannot be separated by a subset of dimension $\leqq n-2$ [7]. For $n=2$, Condition 2 is known to hold [11, p. 110], and in this case we have also the Moore-Kline theorem.

A proof like that of Theorem 6 establishes the condition of the Moore-Kline theorem for the set $T$. Let $\tau$ be an arc component of $R$, and suppose that a point $p$ of $\tau$ other than an endpoint is a limit point of $T-\tau$. Let $\left\{p_{\nu}\right\}$ be a sequence of points of $[T-\tau]$ converging to $p$. The point $p$ cuts $\tau$ into two arcs $\alpha$ and $\beta$. Let $a_{1}, \cdots, a_{m-1}$ be points on $\alpha$ distinct from $p$. The points together with $p$ determine a hyperplane $\pi$. Let $a_{1}$ be the point of $a_{1}, \cdots, a_{m-1}$ such that with the exception of $a_{1}$ and $p$, the arc $a_{1} p$ lies completely on one side of $\pi$. Since $\pi$ contains $m-1$ points of $T$, either

(a) both $a_{1} p$ and $\beta$ are on the same side of $\pi$, in which case the proof is completed as before, or

(b) one arc, call it $\gamma$, and infinitely many points of the sequence lie on the same side of $\pi$. Let $\left\{p_{\nu_{k}}\right\}$ be a subsequence of these points converging to $p$. Then again a sequence of planes $\pi_{\nu_{k}}$ determined by $a_{1}, \cdots, a_{m-1}$ and $p_{v_{k}}$ is such that for $k$ sufficiently large, $\pi_{v_{k}}$ meets the arc $\gamma$, giving $m+1$ points on $\pi_{\nu k}$.

\section{BIBLIOGRAPHY}

1. N. I. Achieser, Vorlesungen über Approximationstheorie, Akademie Verlag, Berlin, 1953. 
2. S. Bernstein, Legons sur les propriêtés extrémales et la meilleure approximation des fonctions analytiques d'une variable réelle, Paris, Gauthier-Villars, 1926.

3. L. N. H. Bunt, Bijdrage tot de theorie der convexe puntverzamelingen, Amsterdam, 1934.

4. W. Fenchel, Über Krümmung und Windung geschlossener Raumkurven, Math. Ann. vol. 101 (1929) pp. 238-252.

5. A. Haar, Die Minkowskische Geometrie und die Annäherung an stetige Funktionen, Math. Ann. vol. 18 (1918) pp. 294-311.

6. O. Hanner and J. Rådström, $A$ generalization of a theorem of Fenchel, Proc. Amer. Math. Soc. vol. 2 (1951) pp. 589-593.

7. W. Hurewicz and H. Wallman, Dimension theory, Princeton University Press, 1941.

8. A. Marchaud, Sur les continus d'ordre borne, Acta Math. vol. 55 (1930) pp. 67115.

9. R. L. Moore and J. R. Kline, On the most general plane closed point-set through which it is possible to pass a simple continuous arc, Ann. of Math. vol. 20 (1919) pp. 218-223.

10. G. Nöbeling, Geometrische (Realitäts) Ordnung und topologische Struktur, J. Reine Angew. Math. vol. 183 (1941) pp. 37-67.

11. G. T. Whyburn, Analytic topology, Amer. Math. Soc. Colloquium Publications, vol. 38, 1942.

12. L. Zippin, The Moore-Kline problem, Trans. Amer. Math. Soc. vol. 34 (1932) pp. 705-721.

University of Pennsylvania 